\title{
An elementary approach to a Girsanov formula and other analytical results on fractional Brownian motions
}

\author{
Ilkka Norros* \\ VTT Information Technology \\ P.O.Box 1202 \\ FIN-02044 VTT \\ Finland \\ ilkka.norros@vtt.fi
}

\author{
Esko Valkeila ${ }^{\dagger}$ \\ Department of Mathematics \\ P.O.Box 4 \\ FIN-00014 University of Helsinki \\ Finland \\ esko.valkeila@helsinki.fi
}

\author{
Jorma Virtamo ${ }^{\ddagger}$ \\ Helsinki University of Technology \\ P.O. Box 3000 \\ FIN-02015 TKK \\ Finland \\ jorma.virtamo@hut.fi
}

Laboratory of Telecommunications Technology

${ }^{\dagger}$ Supported by the Research Grant Committee of the University of Helsinki.

$\ddagger$ The work was done while Jorma Virtamo was with VTT Information Technology. 
Summary The Radon-Nikodym derivative between a centered fractional Brownian motion $Z$ and the same process with constant drift is derived by finding an integral transformation which changes $Z$ to a process with independent increments. A representation of $Z$ through a standard Brownian motion on a finite interval is given. The maximum likelihood estimator of the drift and some other applications are presented.

Keywords: fractional Brownian motion, Gaussian processes, maximum likelihood estimator, prediction, stochastic integration.

\section{Introduction}

Throughout this paper, we denote by $\left(Z_{t}\right)_{t>0}$ a normalized fractional Brownian motion (FBM) with self-similarity parameter $H \in(0,1)$, characterized by the following properties:

(i) $Z_{t}$ has stationary increments;

(ii) $Z_{0}=0$, and $\mathrm{E} Z_{t}=0$ for all $t$;

(iii) $\mathrm{E} Z_{t}^{2}=|t|^{2 H}$ for all $t$;

(iv) $Z_{t}$ is Gaussian;

(v) $Z_{t}$ has continuous sample paths.

We assume that $Z$ is defined on a probability space $(\Omega, \mathcal{F}, P)$ and denote by $\left(\mathcal{F}_{t}\right)_{t \geq 0}$ the filtration (history) generated by $Z$.

This process was originally defined and studied by Kolmogorov (1940) within a Hilbert space framework. In another pioneering paper on FBM, Mandelbrot and Van Ness (1968) defined the process more constructively as the integral

$$
Z_{t}-Z_{s}=c_{H}\left\{\int_{s}^{t}(t-u)^{H-\frac{1}{2}} \mathrm{~d} W_{u}+\int_{-\infty}^{s}\left((t-u)^{H-\frac{1}{2}}-(s-u)^{H-\frac{1}{2}}\right) \mathrm{d} W_{u}\right\},
$$

where $W_{t}$ is the standard Brownian motion. The normalization $\mathrm{E} Z_{1}^{2}=1$ is achieved with the choice

$$
c_{H}=\sqrt{\frac{2 H \Gamma\left(\frac{3}{2}-H\right)}{\Gamma\left(H+\frac{1}{2}\right) \Gamma(2-2 H)}},
$$

where $B$ denotes the beta function

$$
B(\mu, \nu)=\int_{0}^{1} x^{\mu-1}(1-x)^{\nu-1} \mathrm{~d} x=\frac{\Gamma(\mu) \Gamma(\nu)}{\Gamma(\mu+\nu)} .
$$


Note that the choice $H=1 / 2$ gives a standard Brownian motion, and another special case $H=1$ gives a deterministic process with linear paths. In all cases, FBMs are self-similar processes. The sample path assumption (v) above can be strengthened to Hölder continuity (this follows from Kolmogorov's criterion for the continuity of sample paths, see Revuz and Yor 1991):

Theorem 1.1 The sample paths of a continuous fractional Brownian motion with parameter $H$ are, outside a negligible event, Hölder continuous with every exponent $\beta<H$.

When $H \notin\{1 / 2,1\}$, the FBM is neither a Markov process nor a semimartingale (see, e.g., Liptser and Shiryaev 1986, Example 2 of Section 4.9.13). It is, however, a process with a simple structure, and several interesting objects related to it have explicite expressions. For example, a formula for the conditional expectation $\mathrm{E}\left[Z_{T} \mid Z_{s}: s \in[0, t]\right], 0<t<T$, was found by Gripenberg and Norros (1996) (cf. Molchan 1969; see subsection 5.3 below). The aim of this work was to find a counterpart to the Girsanov theorem for Brownian motion, which states, in its simplest form, the following. Let $W$ be the standard Brownian motion, defined on some probability space $(\Omega, \mathcal{F}, P)$, and let $\left(\mathcal{F}_{t}\right)_{t>0}$ be the filtration generated by $W$. Then, for any fixed number $a, L_{t}=e^{a W_{t}-\frac{1}{2} a^{2} t}$ is a martingale with expectation 1 such that, for any $T>0$, with respect to the measure $\mathrm{d} P_{a, T} \doteq L_{T} \mathrm{~d} P,\left(W_{t}\right)_{t \in[0, T]}$ is a standard Brownian motion with drift $a$. Since a Radon-Nikodym derivative process is always a martingale, a central problem is how to construct an appropriate martingale which generates the same filtration as the non-semimartingale $Z$, called here a fundamental martingale.

A classical technique to study the absolute continuity of Gaussian distributions in function spaces is based on reproducing Hilbert space methods. Within this framework, Molchan obtained results on FBM (Molchan 1969; Molchan and Golosov 1969). Recently, stochastic analysis for FBM has been developed by Decreusefond and Üstünel (1997) using Malliavin calculus. In the present paper, we show that many basic results can be obtained more directly with rather elementary arguments and computations.

The paper is organized as follows. In Section 2, heuristic insight is obtained from a discrete time consideration, and some simple technical tools are reviewed. The process $M$ is defined and shown to be a fundamental martingale in Section 3. In Section 4, the desired Girsanov formula is found to be of the same form as in the classical case, with $M$ taking the role of $W$. Four applications of the theory are discussed in Section 5. We note that the study of the fundamental martingale $M$ yields a maximum likelihood estimator of the drift, a representation of $Z$ in terms of standard Brownian motion on a finite interval, a new derivation of the prediction formula of Gripenberg and Norros (1996), and a simple diffusion approximation of the FBM. 


\section{Preliminaries}

\subsection{Heuristics from the discrete time case}

Let $X_{n}, n=1,2, \ldots$, be a centered Gaussian sequence defined on a probability space $(\Omega, \mathcal{F}, P)$. Assume that the covariance matrix $\mathbf{R}_{n}=\operatorname{Cov}\left(X_{1}, \ldots, X_{n}\right)$ is invertible for every $n$. The vector $\mathbf{X}_{n}=\left(X_{1}, \ldots, X_{n}\right)$ has the probability density function $f_{n}(\mathbf{x})=$ $\exp \left(-\frac{1}{2} \mathbf{x}^{T} \mathbf{R}_{n}^{-1} \mathbf{x}\right) / \sqrt{(2 \pi)^{n} \operatorname{Det}\left(\mathbf{R}_{n}\right)}$. Let $a$ be an arbitrary real number. Denote by $\mathbf{1}_{n} \in \mathbb{R}^{n}$ a vector of ones. Then for any $n$, the random variable

$$
L_{n}^{(a)}=\frac{f_{n}\left(\mathbf{X}_{n}-a \mathbf{1}_{n}\right)}{f_{n}\left(\mathbf{X}_{n}\right)}=\exp \left(a \mathbf{1}_{n}^{T} \mathbf{R}_{n}^{-1} \mathbf{X}_{n}-\frac{a^{2}}{2} \mathbf{1}_{n}^{T} \mathbf{R}_{n}^{-1} \mathbf{1}_{n}\right)
$$

has mean 1 , and with respect to the probability $\mathrm{d} P_{n}^{(a)}=L_{n}^{(a)} \mathrm{d} P$, the random variables $X_{1}, \ldots, X_{n}$ have their original covariances but mean $a$. By a general property of RadonNikodym derivatives, $L$ is a martingale. Moreover, the same holds for the sequence $M_{n}=$ $\mathbf{1}_{n}^{T} \mathbf{R}_{n}^{-1} \mathbf{X}_{n}$ appearing in the definition of $L$. Indeed, using the notation

$$
\mathbf{R}_{n+1}=\left[\begin{array}{cc}
\mathbf{R}_{n} & \mathbf{R}_{n 1} \\
\mathbf{R}_{1 n} & r_{n+1}
\end{array}\right], \quad \mathbf{R}_{n+1}^{-1}=\left[\begin{array}{cc}
\mathbf{B}_{n} & \mathbf{B}_{n 1} \\
\mathbf{B}_{1 n} & b_{n+1}
\end{array}\right],
$$

we have the relations

$$
\mathbf{B}_{n}+\mathbf{B}_{n 1} \mathbf{R}_{1 n} \mathbf{R}_{n}^{-1}=\mathbf{R}_{n}^{-1}, \quad \mathbf{B}_{1 n}+b_{n+1} \mathbf{R}_{1 n} \mathbf{R}_{n}^{-1}=0 .
$$

The martingale condition $\mathrm{E}\left[M_{n+1} \mid X_{1}, \ldots, X_{n}\right]=M_{n}$ follows using (2.1) and the identity

$$
\mathrm{E}\left[X_{n+1} \mid X_{1}, \ldots, X_{n}\right]=\mathbf{R}_{1 n} \mathbf{R}_{n}^{-1} \mathbf{X}_{n}
$$

Since $M$ is a Gaussian sequence, the martingale property implies that $M$ has independent increments. (Note that this orthogonalization of the sequence $X$ is not identical to the Gram-Schmidt orthogonalization.) Hence we can write

$$
L_{n}=\exp \left(a M_{n}-\frac{a^{2}}{2}\langle M, M\rangle_{n}\right),
$$

where $\langle M, M\rangle_{n}$ is the angle bracket process of the martingale $M$.

$M_{n}$ can be interpreted as the integral of the deterministic function $w_{n}(i)=\left[\mathbf{1}_{n}^{T} \mathbf{R}_{n}^{-1}\right]_{i}$ with respect to the cumulative process $Z_{k}=\sum_{1}^{k} X_{i}$. Plotting the points $\left(i, w_{n}(i)\right)$ for a discrete process can be used for guessing the expression for the corresponding weight function for a related continuous time process. In the case of fractional Brownian motion $Z$ with $H>1 / 2$, the discrete time weight function $w_{n}(i)$ for $X_{i}=Z_{i}-Z_{i-1}$ is seen to have the form of the letter $\mathrm{U}$. This suggests that in the continuous time, the process $M_{t}=\int_{0}^{t} w(t, s) \mathrm{d} Z_{s}$, where $w(t, s)=$ const $\cdot s^{-\alpha}(t-s)^{-\alpha}$, would be a martingale with certain $\alpha$. Further, the representation (1.1) suggests that $\alpha=H-\frac{1}{2}$. Having guessed the result, it remains to prove it and to compute the constant. Tools for doing this in an elementary way are identified in the following subsections. 


\subsection{Remarks on integration}

Integration with respect to $Z$ plays a central role below. Since $Z$ is not a semimartingale, we refer to the integration theory of Gaussian processes (see, e.g., Huang and Cambanis 1978) instead of the more usual martingale approach. In fact, we need to consider deterministic integrands only, and the technical framework can be described briefly. Both $L^{2}$ and pathwise approaches are possible.

For $H>1 / 2$, let $\Gamma$ denote the integral operator

$$
\Gamma f(t)=H(2 H-1) \int_{0}^{\infty} f(s)|s-t|^{2 H-2} \mathrm{~d} s,
$$

and define the inner product

$$
\langle f, g\rangle_{\Gamma}=\left\langle\langle f, \Gamma g\rangle=H(2 H-1) \int_{0}^{\infty} \int_{0}^{\infty} f(s) g(t)|s-t|^{2 H-2} \mathrm{~d} s \mathrm{~d} t\right.
$$

where $\langle\cdot\rangle\rangle$ denotes the usual inner product of $L^{2}[0, \infty)$. Denote by $L_{\Gamma}^{2}$ the space of equivalence classes of measurable functions $f$ such that $\left\langle\langle f, f\rangle_{\Gamma}\langle\infty\right.$. Now, it is easy to check that the association

$$
Z_{t} \mapsto 1_{[0, t)}
$$

can be extended to an isometry between the Gaussian space generated by the random variables $Z_{t}, t \geq 0$, as the smallest closed linear subspace of $L^{2}(\Omega, \mathcal{F}, P)$ containing them, and the function space $L_{\Gamma}^{2}$. For $f \in L_{\Gamma}^{2}$, the integral $\int_{0}^{\infty} f(t) \mathrm{d} Z_{t}$ can now be defined as the image of $f$ in this isometry.

For $H<1 / 2$, the integral in the above definition of $\Gamma$ diverges, and we have to define the operator in another way. In this case, an appropriate definition of $\Gamma$ is

$$
\Gamma f(t)=H \int_{0}^{\infty}|t-s|^{2 H-1} \operatorname{sgn}(t-s) \mathrm{d} f(s) .
$$

We interprete $f(0-)=0$ so that, for example, the indicator function $1_{[0, t)}$ is identified with the signed measure $\delta_{0}-\delta_{t}$. It is again easy to check that $Z_{t} \mapsto 1_{[0, t)}$ defines an isometry. Below we apply $\Gamma$ only to explicitly given functions $f$.

If $f$ has bounded variation, the integral $\int_{0}^{T} f(t) \mathrm{d} Z_{t}(\omega)$ can be defined $\omega$ by $\omega$ as a limit of Riemann sums, and it is easy to see that the integral obtained coincides with the $L^{2}$ integral almost surely. The convergence of these Riemann sums is equivalent to that of the usual Riemann-Stieltjes integral appearing at the right hand side of the integration by parts formula

$$
\int_{0}^{T} f(t) \mathrm{d} Z_{t}(\omega)=f(T) Z_{T}(\omega)-f(0) Z_{0}(\omega)-\int_{0}^{T} Z_{t}(\omega) \mathrm{d} f(t),
$$

and thus guaranteed by the continuity of the sample paths of $Z$. 
Much stronger results can be obtained using the Hölder continuity of most sample paths of $Z$ (Theorem 1.1). In fact, all processes considered in this paper, obtained from each other by integrating fractional powers or a little more complicated integrands with respect to a Gaussian process, can be defined by pathwise integration outside a set of measure zero. The basic idea why this is possible is presented in the following elementary lemma.

Lemma 2.1 Assume that the function $f:[0, T] \rightarrow \mathbb{R}$ with $f(0)=0$ is Hölder continuous with exponent $\beta \in(0,1)$. Let $\gamma \in(-\beta,-\beta+1)$. Then the function

$$
\begin{aligned}
g(t) & =\int_{0}^{t}(t-u)^{\gamma} \mathrm{d} f(u) \\
& \doteq \lim _{\epsilon \rightarrow 0}\left(\epsilon^{\gamma}(f(t-\epsilon)-f(t))+t^{\gamma} f(t)+\gamma \int_{0}^{t-\epsilon}(f(u)-f(t))(t-u)^{\gamma-1} \mathrm{~d} u\right)
\end{aligned}
$$

is well defined, finite and Hölder continuous with exponent $\beta+\gamma$.

Proof By assumption, there is a number $K>0$ such that $|f(t)-f(s)| \leq K|t-s|^{\beta}$ for $t, s \in[0, T]$. Note first that for $a<b<v$ and for any $\alpha$ we have

$$
\left|\int_{a}^{b}(v-u)^{\alpha} \mathrm{d} f(u)\right| \leq\left(K+\left|\frac{\alpha}{\alpha+\beta}\right|\right)\left((v-a)^{\alpha+\beta}+(v-b)^{\alpha+\beta}\right) .
$$

It is easy to see that the limit in the definition of $g(t)$ exists and is finite. To show the Hölder continuity, write for $s<t$ the difference $g(t)-g(s)$ as

$$
\begin{aligned}
g(t)-g(s) & =\int_{s}^{t}(t-u)^{\gamma} \mathrm{d} f(u)+\int_{0}^{s}\left((t-u)^{\gamma}-(s-u)^{\gamma}\right) \mathrm{d} f(u) \\
& =\int_{s}^{t}(t-u)^{\gamma} \mathrm{d} f(u)+\int_{0}^{s}\left(\gamma \int_{s}^{t}(v-u)^{\gamma-1} \mathrm{~d} v\right) \mathrm{d} f(u) \\
& =\int_{s}^{t}(t-u)^{\gamma} \mathrm{d} f(u)+\lim _{\epsilon \rightarrow 0} \int_{0}^{s-\epsilon}\left(\gamma \int_{s}^{t}(v-u)^{\gamma-1} \mathrm{~d} v\right) \mathrm{d} f(u) \\
& =\int_{s}^{t}(t-u)^{\gamma} \mathrm{d} f(u)+\lim _{\epsilon \rightarrow 0} \int_{s}^{t}\left(\gamma \int_{0}^{s-\epsilon}(v-u)^{\gamma-1} \mathrm{~d} f(u)\right) \mathrm{d} v \\
& =\int_{s}^{t}(t-u)^{\gamma} \mathrm{d} f(u)+\int_{s}^{t}\left(\gamma \int_{0}^{s}(v-u)^{\gamma-1} \mathrm{~d} f(u)\right) \mathrm{d} v,
\end{aligned}
$$

where the change of the order of integration is easily justified since the integrand is bounded. Taking the limit under the integration sign is allowed by the dominated convergence theorem, using (2.3) with $\alpha=\gamma-1$. Finally, apply (2.3) to the both terms to obtain the desired inequality

$$
|g(t)-g(s)| \leq K^{\prime}|t-s|^{\beta+\gamma}
$$

with a certain constant $K^{\prime}$. 
It is straightforward to extend the above arguments to the somewhat more complicated integrands with fractional power singularities which appear throughout this paper. For more general theory, we refer to the recent paper by Feyel and de La Pradelle (1996) and the references therein.

\subsection{Equations for integrals of fractional powers}

The following relations between integrals of fractional powers play a central role in this paper.

Lemma 2.2 (i) For $\mu, \nu>0, c>1$, we have

$$
\int_{0}^{1} t^{\mu-1}(1-t)^{\nu-1}(c-t)^{-\mu-\nu} \mathrm{d} t=c^{-\nu}(c-1)^{-\mu} B(\mu, \nu)
$$

(ii) For $\mu \in \mathbb{R}, \nu>-1, c>1$, we have

$$
\int_{1}^{c} t^{\mu}(t-1)^{\nu} \mathrm{d} t=\int_{0}^{1-1 / c} s^{\nu}(1-s)^{-\mu-\nu-2} \mathrm{~d} s .
$$

(iii) Assume that $\mu, \nu>0$ and $c>1$. Then

$$
\begin{aligned}
& \int_{0}^{1} t^{\mu-1}(1-t)^{\nu-1}(c-t)^{-\mu-\nu+1} \mathrm{~d} t \\
= & B(\mu, 1-\mu)-(\mu+\nu-1) B(\mu, \nu) \int_{0}^{1-1 / c} s^{-\mu}(1-s)^{\mu+\nu-2} \mathrm{~d} s \\
= & (\mu+\nu-1) B(\mu, \nu) c^{-\nu+1} \int_{0}^{1} s^{\mu+\nu-2}(c-s)^{-\mu} \mathrm{d} s,
\end{aligned}
$$

where the second form requires additionally that $\mu<1$ and the third that $\mu+\nu>1$.

(iv) For $\mu \in(0,1), x \in(0,1)$, we have

$$
\int_{0}^{1} t^{-\mu}(1-t)^{-\mu}|x-t|^{2 \mu-1} \mathrm{~d} t=B(\mu, 1-\mu) .
$$

Proof Assertions (i) and (ii) follow with the substitutions $t=c s /(c-1+s)$ and $t=$ $1 /(1-s)$, respectively. (iii) is a little more tricky. Assume that $\mu<1$ - this can be relaxed 
for the equality between the first and last form since both are analytic in $\mu$ for $\mu>0$. Now,

$$
\begin{aligned}
& \int_{0}^{1} t^{\mu-1}(1-t)^{\nu-1}(c-t)^{-\mu-\nu+1} \mathrm{~d} t \\
= & \int_{0}^{1} t^{\mu-1}(1-t)^{\nu-1}\left[(1-t)^{-\mu-\nu+1}+(-\mu-\nu+1) \int_{1}^{c}(v-t)^{-\mu-\nu} \mathrm{d} v\right] \mathrm{d} t \\
= & B(\mu, 1-\mu)-(\mu+\nu-1) \int_{1}^{c}\left[\int_{0}^{1} t^{\mu-1}(1-t)^{\nu-1}(v-t)^{-\mu-\nu} \mathrm{d} t\right] \mathrm{d} v \\
\stackrel{(\mathrm{i})}{=} & B(\mu, 1-\mu)-(\mu+\nu-1) B(\mu, \nu) \int_{1}^{c} v^{-\nu}(v-1)^{-\mu} \mathrm{d} v \\
\stackrel{(\mathrm{ii})}{=} & B(\mu, 1-\mu)-(\mu+\nu-1) B(\mu, \nu) \int_{0}^{1-1 / c} s^{-\mu}(1-s)^{\mu+\nu-2} \mathrm{~d} s \\
= & B(\mu, 1-\mu)-(\mu+\nu-1) B(\mu, \nu)\left[B(1-\mu, \mu+\nu-1)-\int_{0}^{1 / c} s^{\mu+\nu-2}(1-s)^{-\mu} \mathrm{d} s\right] \\
= & (\mu+\nu-1) B(\mu, \nu) \int_{0}^{1 / c} s^{\mu+\nu-2}(1-s)^{-\mu} \mathrm{d} s,
\end{aligned}
$$

where the last step follows from the identity

$$
(\mu+\nu-1) B(\mu, \nu) B(1-\mu, \mu+\nu-1)=B(\mu, 1-\mu) .
$$

Finally, (iv) follows easily from (iii) and (2.4).

Many results below are proved very simply using the following fact.

Proposition 2.3 Let $w(t, s)$ be the function

$$
w(t, s)= \begin{cases}c_{1} s^{\frac{1}{2}-H}(t-s)^{\frac{1}{2}-H}, & \text { for } s \in(0, t), \\ 0, & \text { for } s \notin(0, t),\end{cases}
$$

where

$$
c_{1}=\left[2 H B\left(\frac{3}{2}-H, H+\frac{1}{2}\right)\right]^{-1} .
$$

Then

$$
\Gamma w(t, \cdot)(s)= \begin{cases}1, & \text { for } s \in[0, t), \\ \frac{\left(H-\frac{1}{2}\right) s^{H-\frac{1}{2}}}{\left(\frac{3}{2}-H\right) B\left(H+\frac{1}{2}, 2-2 H\right)} \int_{0}^{t} u^{1-2 H}(s-u)^{H-\frac{3}{2}} \mathrm{~d} u, & \text { for } s>t .\end{cases}
$$


Proof Consider first the case $H>1 / 2$. For $s \in[0, t]$, the assertion is a direct consequence of item (iv) of Lemma 2.2, with the choice $\mu=H-\frac{1}{2}$. For $s>t$, the result is obtained using item (iii) of the same lemma.

For $H<1 / 2$, we have to evaluate

$$
\Gamma w(t, \cdot)(s)=H\left(\frac{1}{2}-H\right) c_{1} \int_{0}^{t}|s-u|^{2 H-1} \operatorname{sgn}(s-u) u^{-\frac{1}{2}-H}(t-u)^{-\frac{1}{2}-H}(t-2 u) \mathrm{d} u .
$$

For $s \in[0, t)$, this expression is divided into 4 integrals which are then computed with item (i) and with the both forms given in item (iii) of Lemma 2.2. Finally, the result for $s>t$ is obtained applying first items (i) and (iii) of Lemma 2.2 and then the identity

$$
(1-2 H) s t^{H-\frac{1}{2}} \int_{0}^{t} u^{-2 H}(s-u)^{H-\frac{3}{2}} \mathrm{~d} u=\left(\frac{s-t}{t}\right)^{H-\frac{1}{2}}-\left(H-\frac{1}{2}\right) t^{H-\frac{1}{2}} \int_{0}^{t} u^{1-2 H}(s-u)^{H-\frac{3}{2}} \mathrm{~d} u .
$$

The latter can be derived using item (ii) of Lemma 2.2 once in both directions, integrating by parts between.

Note that for $H<1 / 2$, the function $\Gamma w(t, \cdot)$ has a singularity at $t$.

\section{The fundamental martingale $M$}

As noted in the Introduction, the fractional Brownian motion is not a semimartingale. Its paths are continuous with locally unbounded variation but with zero quadratic variation. However, we can prove the following. Let $w(t, s)$ be the function appearing in Proposition 2.3 .

Theorem 3.1 The centered Gaussian process

$$
M_{t}=\int_{0}^{t} w(t, s) \mathrm{d} Z_{s}
$$

has independent increments and variance function

$$
\mathrm{E} M_{t}^{2}=c_{2}^{2} t^{2-2 H}
$$

where

$$
c_{2}=\frac{c_{H}}{2 H \sqrt{2-2 H}}
$$

In particular, $M$ is a martingale. 
Proof By Proposition 2.3, we have for $s<t$ :

$$
\begin{aligned}
\operatorname{Cov}\left(M_{s}, M_{t}\right) & =\langle w(s, \cdot), \Gamma w(t, \cdot)\rangle\rangle \\
& =\left\langle w(s, \cdot), 1_{[0, t]}\right\rangle \\
& =\int_{0}^{s} w(s, u) \mathrm{d} u \\
& =c_{1} B\left(\frac{3}{2}-H, \frac{3}{2}-H\right) s^{2-2 H} \\
& =c_{2}^{2} s^{2-2 H} .
\end{aligned}
$$

The end result is independent of $t$ which shows that $M$ has uncorrelated and thus, since it is a Gaussian process, independent increments.

Note that the process

$$
W_{t}=\frac{2 H}{c_{H}} \int_{0}^{t} s^{H-\frac{1}{2}} \mathrm{~d} M_{s}
$$

is a standard Brownian motion.

The covariance $\operatorname{Cov}\left(Z_{s}, M_{t}\right)$ has a particularly simple expression when $s \leq t$ :

Proposition 3.2 For all $0 \leq s \leq t$, we have

$$
\operatorname{Cov}\left(Z_{s}, M_{t}\right)=s
$$

As a consequence, the increment $M_{t}-M_{s}$ is independent of $\mathcal{F}_{s}$.

Proof By Proposition 2.3, $\operatorname{Cov}\left(Z_{s}, M_{t}\right)=\left\langle\left\langle 1_{[0, s]}, \Gamma w(t, \cdot)\right\rangle=\left\langle\left\langle 1_{[0, s]}, 1_{[0, t]}\right\rangle\right\rangle=s\right.$.

It turns out, however, that the covariance $\operatorname{Cov}\left(Z_{s}, M_{t}\right)$ does not have a nice expression for $s>t$, and it is easier to proceed by considering, instead of $Z$, the process

$$
Y_{t} \doteq \int_{0}^{t} s^{\frac{1}{2}-H} \mathrm{~d} Z_{s} .
$$

It is obvious that we have the inverse relationship $Z_{t}=\int_{0}^{t} s^{H-\frac{1}{2}} \mathrm{~d} Y_{s}$; in particular, $Y$ generates the same filtration $\left(\mathcal{F}_{t}\right)$ as $Z$. 
Proposition 3.3 For $Y$ defined by (3.2), we have

$$
\mathrm{E}_{t} Y_{T}= \begin{cases}\frac{c_{H}^{2}}{2 H} \int_{0}^{t}(T-s)^{H-\frac{1}{2}} s^{1-2 H} \mathrm{~d} s, & t<T \\ \frac{T^{\frac{3}{2}-H}}{\frac{3}{2}-H}, & t \geq T .\end{cases}
$$

Proof The case $t \geq T$ follows from the other case by noting that, by Proposition 3.2, the increments of $M$ after time $T$ are independent of $Y_{T}$. For $t<T$, the result follows from Proposition 2.3. Note first that

$$
\mathrm{E} M_{t} Y_{t}=\left\langle s^{\frac{1}{2}-H} 1_{[0, t)}(s), \Gamma w(t, \cdot)(s)\right\rangle=\int_{0}^{t} s^{\frac{1}{2}-H} \mathrm{~d} s=\frac{t^{\frac{3}{2}-H}}{\frac{3}{2}-H} .
$$

Second, a short computation shows that

$$
\mathrm{E}_{t}\left(Y_{T}-Y_{t}\right)=\left\langle\left\langle s^{\frac{1}{2}-H} 1_{[t, T)}(s), \Gamma w(t, \cdot)(s)\right\rangle=\frac{c_{H}^{2}}{2 H} \int_{0}^{t} u^{1-2 H}(T-u)^{H-\frac{1}{2}} \mathrm{~d} u-\frac{t^{\frac{3}{2}-H}}{\frac{3}{2}-H} .\right.
$$

Only a little remains to prove that $M$ is indeed a fundamental martingale in the above mentioned sense that it generates the same filtration as $Z$. This is shown by deriving a representation of the process $Y$ in terms of $M$.

Theorem 3.4 The martingale $M$ generates, up to sets of measure zero, the same filtration as $Z$. The same holds for the related processes $W$ and $Y$, defined in (3.1) and (3.2), respectively. The process $Y$ has the integral representation

$$
Y_{T}=2 H \int_{0}^{T}(T-t)^{H-\frac{1}{2}} \mathrm{~d} M_{t}
$$

and we have the prediction formula

$$
\mathrm{E}\left[Y_{T} \mid \mathcal{F}_{t}\right]=2 H \int_{0}^{t}(T-s)^{H-\frac{1}{2}} \mathrm{~d} M_{s}
$$

Proof Denote by $\left(\mathcal{F}_{t}^{M}\right)_{t \geq 0}$ the filtration generated by $M$. We first show that for $t \leq T$,

$$
\mathrm{E}\left[Y_{T} \mid \mathcal{F}_{t}^{M}\right]=2 H \int_{0}^{t}(T-s)^{H-\frac{1}{2}} \mathrm{~d} M_{s} \doteq \hat{Y}_{T \mid t} .
$$


It is enough to show that the difference $Y_{T}-\hat{Y}_{T \mid t}$ is orthogonal to $M_{s}$ for $s \leq t$. Further, since $M$ has independent increments, we have $\mathrm{E} M_{s} \hat{Y}_{T \mid t}=\mathrm{E} M_{s} \hat{Y}_{T \mid s}$ for $s<t$, so that we only have to show that $\mathrm{E}_{t} \hat{Y}_{T \mid t}=\mathrm{E} M_{t} Y_{T}$. Since

$$
\mathrm{E} M_{t} \hat{Y}_{T \mid t}=2 H \int_{0}^{t}(T-s)^{H-\frac{1}{2}} \mathrm{~d}\langle M, M\rangle_{s}=\frac{c_{H}^{2}}{2 H} \int_{0}^{t}(T-s)^{H-\frac{1}{2}} s^{1-2 H} \mathrm{~d} s,
$$

this follows from Proposition 3.3.

In order to see that in fact $\hat{Y}_{T \mid T}=Y_{T}$ a.s., we only have to check that $\mathrm{E}\left(\hat{Y}_{T \mid T}\right)^{2}=\mathrm{E} Y_{T}^{2}$. Indeed, we have

$$
\mathrm{E}\left(\hat{Y}_{T \mid T}\right)^{2}=(2 H)^{2} \int_{0}^{T}(T-t)^{2 H-1} \mathrm{~d}\langle M, M\rangle_{t}=c_{H}^{2} B(2 H, 2-2 H) T,
$$

and short computations, for the cases $H<1 / 2$ and $H>1 / 2$ separately, show that we also have $\mathrm{E} Y_{T}^{2}=c_{H}^{2} B(2 H, 2-2 H) T$. Now, it is easy to see that for any $t, \mathcal{F}_{t}=\mathcal{F}_{t}^{Y}=\mathcal{F}_{t}^{M}=\mathcal{F}^{W}$ up to sets of measure zero.

Remark 3.5 The definition of $M$ in Theorem 3.1 can be written as

$$
c_{1} \int_{0}^{t}(t-s)^{\frac{1}{2}-H} \mathrm{~d} Y_{s}=M_{t}
$$

This is a generalized Abel's integral equation with respect to the process $Y_{t}$. A formal solution yields

$$
Y_{t}=\frac{1}{c_{1} B\left(H+\frac{1}{2}, \frac{3}{2}-H\right)} \int_{0}^{t}(t-s)^{H-\frac{1}{2}} \mathrm{~d} M_{s}=2 H \int_{0}^{t}(t-s)^{H-\frac{1}{2}} \mathrm{~d} M_{s} .
$$

Thus, we could alternatively have proved Theorem 3.4 by solving these pathwise integral equations.

Remark 3.6 It is well known that all right continuous square integrable $\left(\mathcal{F}_{t}^{W}\right)$-martingales can be expressed as stochastic integrals with respect to $W$. Since the filtrations $\left(\mathcal{F}_{t}^{W}\right)$ and $\left(\mathcal{F}_{t}\right)$ coincide up to sets of measure zero, it follows that all right continuous square integrable $\left(\mathcal{F}_{t}\right)$-martingales can be expressed as stochastic integrals with respect to $W$. Since $W$ itself is obtained from $Z$ with an integral transform, one could expect that all the above mentioned martingales could also be represented as suitably defined stochastic integrals with respect to $Z$. It seems, however, that it is still not clear how the theory of stochastic integration with respect to non-semimartingales should be built. 


\section{The Girsanov formula}

Denote by $\mathcal{E}(M)$ the stochastic exponent of $M$,

$$
\mathcal{E}_{t}(M) \doteq \exp \left(M_{t}-\frac{1}{2}\langle M, M\rangle_{t}\right)
$$

By a standard result of martingale theory, $\mathcal{E}_{t}(M)$ is a martingale with expectation 1. Let us restrict to a finite time interval $[0, T]$. For $a \in \mathbb{R}$, let $P_{a}$ be the probability on $\left(\Omega, \mathcal{F}_{T}, P\right)$ defined by the relation

$$
\left(\frac{\mathrm{d} P_{a}}{\mathrm{~d} P}\right)_{\mathcal{F}_{T}} \doteq \mathcal{E}_{T}(a M) .
$$

(It is easy to see that if the measure $P_{a}$ can be extended to the $\sigma$-algebra $\mathcal{F}_{\infty}$, this extension is singular with respect to $P$.)

In this section we prove the Girsanov type result that $\mathcal{E}_{T}(a M)$ is in fact the likelihood ratio between the two hypotheses

$H_{0}$ With respect to the measure $P$ the process $X$ is a fractional Brownian motion with index $H$, i.e. $X_{t}=Z_{t}$.

$H_{a}$ With respect to the measure $P_{a}$ the process $X$ is a fractional Brownian motion with constant drift $a$, i.e. $X_{t}=Z_{t}+a t$.

Theorem 4.1 With respect to the measure $P_{a}$, the process $Z$ is a fractional Brownian motion with drift $a$, i.e., the distribution of $Z$ with respect to $P_{a}$ is the same as the distribution of $Z_{t}+$ at with respect to $P=P_{0}$.

Proof It is sufficient to prove that the finite dimensional distributions of $Z$ with respect to the measure $P_{a}$ are those of a fractional Brownian motion with a drift $a$.

So fix $t_{i} \in[0, T], i=1, \cdots, n$, and put $\tau^{t}=\left(t_{1}, \cdots, t_{n}\right)$. We have to show that

$$
\left(X_{t_{1}}, \cdots, X_{t_{n}}\right) \stackrel{P_{a}}{\sim} N(a \tau, \Sigma)
$$

where $\Sigma \doteq\left(\operatorname{Cov}_{0}\left(Z_{t_{i}}, Z_{t_{j}}\right)\right)_{i, j=1, \ldots, n}$.

Pick $\alpha^{t}=\left(\alpha_{1}, \cdots, \alpha_{n}\right)$ and note that

$$
E_{a} \exp \left(\sum_{i=1}^{n} \alpha_{i} Z_{t_{i}}\right)=E_{0} \exp \left(\sum_{i=1}^{n} \alpha_{i} Z_{t_{i}}+a M_{T}-\frac{a^{2}}{2}\langle M, M\rangle_{T}\right) .
$$


With respect to the measure $P_{0}$ the random variable $U \doteq \sum_{i=1}^{n} \alpha_{i} Z_{t_{i}}+a M_{T}$ is a Gaussian random variable with mean $E_{0} U=0$ and variance

$$
E_{0} U^{2}=E_{0}\left(a M_{T}\right)^{2}+E_{0}\left(\sum_{i=1}^{n} \alpha_{i} Z_{t_{i}}\right)^{2}+2 \sum_{i=1}^{n} \operatorname{Cov}_{0}\left(a M_{T}, \alpha_{i} Z_{t_{i}}\right)
$$

Now, by Proposition 3.2,

$$
\operatorname{Cov}_{0}\left(a M_{T}, \alpha_{i} Z_{t_{i}}\right)=\alpha_{i} a \operatorname{Cov}_{0}\left(M_{t_{i}}, Z_{t_{i}}\right)=\alpha_{i} a t_{i}
$$

Hence

$$
E_{0} U^{2}=a^{2}\langle M, M\rangle_{T}+\alpha^{t} \Sigma \alpha+2 \sum_{i=1}^{n} \alpha_{i} a t_{i}
$$

and, since $U$ is Gaussian,

$$
E_{0} \exp (U)=\exp \left(\frac{a^{2}\langle M, M\rangle_{T}+2 \alpha^{t} a \tau+\alpha^{t} \Sigma \alpha}{2}\right)
$$

Thus we have shown that

$$
E_{a} \exp \left(\sum_{i=1}^{n} \alpha_{i} Z_{t_{i}}\right)=\exp \left(a \alpha^{t} \tau+\frac{\alpha^{t} \Sigma \alpha}{2}\right)
$$

i.e., under the measure $P_{a}$ the finite dimensional distribution of the vector $\left(Z_{t_{1}}, \cdots, Z_{t_{n}}\right)$ has a multivariate normal distribution with mean $a \tau$ and covariance that of fractional Brownian motion.

\section{Applications}

\subsection{Maximum likelihood estimation of the drift}

Consider again the $P$-martingale $M$ and define a measure $P_{a}$ by

$$
\mathrm{d} P_{a} \doteq \mathcal{E}(a M) \mathrm{d} P_{0}
$$

It is readily found by differentiating $\mathcal{E}(a M)$ with respect to $a$ that the maximum likelihood estimator $\hat{a}_{T}$, based on observation of the process on an interval $[0, T]$, is given by the formula

$$
\hat{a}_{T}=\frac{M_{T}}{\langle M, M\rangle_{T}} .
$$

The basic properties of this estimator are given in the next theorem. 
Theorem 5.1 The maximum likelihood estimator $\hat{a}_{t}$ is unbiased and normally distributed:

$$
\hat{a}_{t} \stackrel{d\left(P_{a}\right)}{=} N\left(a, 1 /\langle M, M\rangle_{t}\right)=N\left(a, \frac{1}{c_{2}^{2} t^{2-2 H}}\right) .
$$

It is also consistent: for all $a \in \mathbb{R}$,

$$
\lim _{t \rightarrow \infty} \hat{a}_{t} \stackrel{P_{a}-\text { a.s. }}{=} a \text {. }
$$

Moreover, we have the following law of the iterated logarithm:

$$
\limsup _{t} \frac{\sqrt{A_{t}}\left|\hat{a}_{t}-a\right|}{\sqrt{2 \log \log A_{t}}}=1 P_{a} \text {-a.s. }
$$

where $A \doteq\langle M, M\rangle$.

Proof It is well known that the so-called local characteristics of the $P_{0}$-martingale $M$ with respect to the measure $P_{a}$ are $(a\langle M, M\rangle,\langle M, M\rangle, 0)$ (see, e.g., Jacod and Shiryaev 1987, Theorem III.3.24). This means that the process $M-a\langle M, M\rangle$ is a $P_{a}$-martingale.

The consistency of $\hat{a}$ is now almost obvious:

$$
\hat{a}_{t}-a=\frac{M_{t}-a\langle M, M\rangle_{t}}{\langle M, M\rangle_{t}}
$$

where the process $M-a\langle M, M\rangle$ is $P_{a}$-martingale; so the consistency follows from the strong law of large numbers for martingales (see, e.g., Liptser and Shiryaev 1986, Theorem 2.6.10). Finally, since the deviation $\hat{a}-a$ is obtained from a continuous Gaussian martingale, the last assertion follows from Corollary 1.1.12 of Revuz and Yor 1991.

An obvious choice for an estimator of the drift would of course be the mean $Z_{T} / T$ which is an unbiased estimator with variance $t^{2 H-2}$. Thus, the variances of these two unbiased estimators differ by the constant multiplier $1 / c_{2}^{2}$. Now, the function $H \mapsto 1 / c_{2}(H)$ has an interesting shape (see Figure 5.1). For $H \geq 1 / 2,1 / c_{2}(H)$ is very close to one, so that the maximum likelihood estimator gives only negligible improvement over the simple mean. On the other hand, $1 / c_{2}(H)$ is much less than one for small $H$, and using the maximum likelihood estimator can give a clear advantage. The corresponding fact has been observed in a more general time series context by Samarov and Taqqu (1988).

\subsection{Representation of $Z$ through a Brownian motion on a finite interval}

As already noted in Section 3, the process

$$
W_{t}=\frac{2 H}{c_{H}} \int_{0}^{t} s^{H-\frac{1}{2}} \mathrm{~d} M_{s}
$$




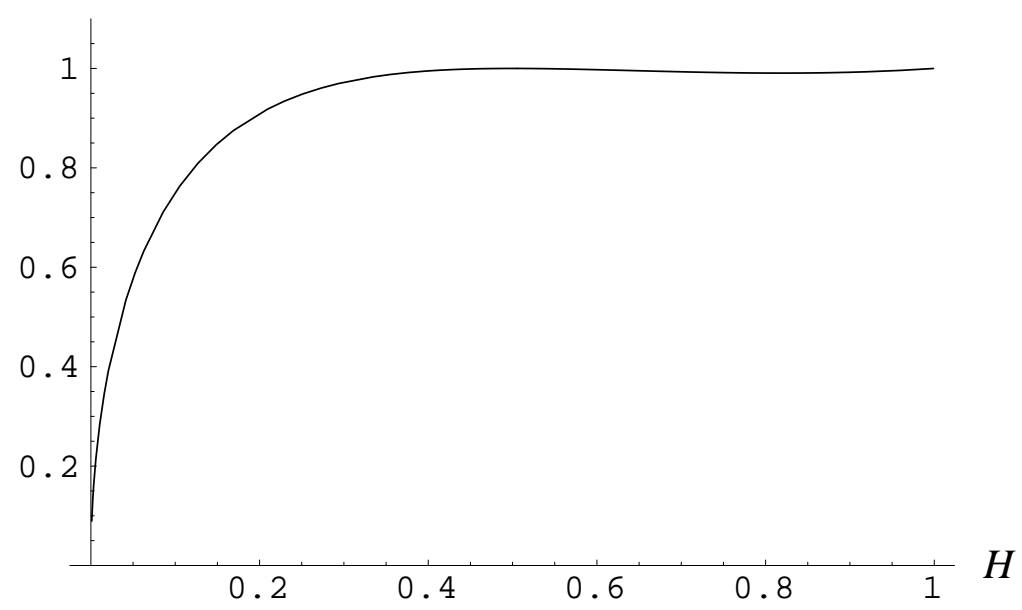

Figure 5.1: The number $1 / c_{2}$ as a function of $H$.

is a standard Brownian motion. We also have the inverse relationship

$$
M_{t}=\frac{c_{H}}{2 H} \int_{0}^{t} s^{\frac{1}{2}-H} \mathrm{~d} W_{s} .
$$

Thus, the results of Section 3 give a sequence of simple representation formulae which allow us to proceed from process to process in the order $W \rightarrow M \rightarrow Y \rightarrow Z$. It is now straightforward to combine these steps into a single integral transformation which brings $W$ to $Z$ :

Theorem 5.2 The process $Z$ has the following integral representation in terms of $W$ :

$$
Z_{t}=\int_{0}^{t} z(t, s) \mathrm{d} W_{s},
$$

where

$$
z(t, s)=c_{H}\left[\left(\frac{t}{s}\right)^{H-\frac{1}{2}}(t-s)^{H-\frac{1}{2}}-\left(H-\frac{1}{2}\right) s^{\frac{1}{2}-H} \int_{s}^{t} u^{H-\frac{3}{2}}(u-s)^{H-\frac{1}{2}} \mathrm{~d} u\right] .
$$

For $H>\frac{1}{2}$ we have also the slightly simpler expression

$$
\begin{aligned}
z(t, s) & =\left(H-\frac{1}{2}\right) c_{H} s^{\frac{1}{2}-H} \int_{s}^{t} u^{H-\frac{1}{2}}(u-s)^{H-\frac{3}{2}} \mathrm{~d} u \\
& =c_{H}(t-s)^{H-\frac{1}{2}}{ }_{2} F_{1}\left(\frac{1}{2}-H, H-\frac{1}{2}, H+\frac{1}{2}, 1-t / s\right),
\end{aligned}
$$

where ${ }_{2} F_{1}$ is the Gauss hypergeometric function. Moreover, we have the prediction formula

$$
\hat{Z}_{T \mid t} \doteq \mathrm{E}\left[Z_{T} \mid \mathcal{F}_{t}\right]=\int_{0}^{t} z(T, s) \mathrm{d} W_{s} .
$$


Proof Denote again $\alpha=H-\frac{1}{2}$. Since $Y_{s}=c_{H} \int_{0}^{s} u^{-\alpha}(s-u)^{\alpha} \mathrm{d} W_{u}$, we have

$$
\begin{aligned}
Z_{t} & =\int_{0}^{t} s^{\alpha} \mathrm{d} Y_{s} \\
& =t^{\alpha} Y_{t}-\alpha \int_{0}^{t} s^{\alpha-1} Y_{s} \mathrm{~d} s \\
& =c_{H} t^{\alpha} \int_{0}^{t} u^{-\alpha}(t-u)^{\alpha} \mathrm{d} W_{u}-c_{H} \alpha \int_{0}^{t} \mathrm{~d} s s^{\alpha-1} \int_{0}^{s} \mathrm{~d} W_{u} u^{-\alpha}(s-u)^{\alpha} .
\end{aligned}
$$

The assertion now follows by changing the order of integration. The alternative form for $H>\frac{1}{2}$ is obtained by integrating by parts. Finally, the prediction formula (5.2) is an immediate consequence of the fact that its right hand side is an $\mathcal{F}$-martingale.

Remark 5.3 The better known representation (1.1) can be obtained from Theorem 5.2 as a limit result.

\subsection{The prediction formula for FBM}

Gripenberg and Norros (1996) showed (with slightly different notation; cf. Molchan 1969):

Theorem 5.4 For $H>\frac{1}{2}$, we have the prediction formula

$$
\mathrm{E}\left[Z_{T} \mid Z_{s}, s \in[0, t]\right]=Z_{t}+\int_{0}^{t} \Psi_{T}(t, s) \mathrm{d} Z_{s},
$$

where the weight function $\Psi_{T}(t, s)$ has the expression

$$
\Psi_{T}(t, s)=\frac{\sin \left(\pi\left(H-\frac{1}{2}\right)\right)}{\pi} s^{-H+\frac{1}{2}}(t-s)^{-H+\frac{1}{2}} \int_{t}^{T} \frac{u^{H-\frac{1}{2}}(u-t)^{H-\frac{1}{2}}}{u-s} \mathrm{~d} u .
$$

The theory developed in this paper offers a new way to derive this result. We only have to transform the prediction formula (5.2) into an integral with respect to $Z$. To transform

the result to the form given above requires, however, a rather long computation, and we skip the details here.

\subsection{Approximation of $Z$ with a simple diffusion}

By Proposition 3.2, the correlation coefficient between $Z_{t}$ and $M_{t}$ is, for any $t \geq 0$,

$$
\operatorname{Corr}\left(Z_{t}, M_{t}\right)=\frac{t}{t^{H} \cdot c_{2} t^{1-H}}=\frac{1}{c_{2}} .
$$


Now, the number $1 / c_{2}=1 / c_{2}(H)$ is very close to one when $H \in\left(\frac{1}{2}, 1\right)$, with equality at the endpoints and a minimum value of about 0.99 . Thus, one can use the diffusion process

$$
X_{t} \doteq \frac{t^{2 H-1}}{c_{2}} M_{t}
$$

as an approximation to $Z$ for some practical purposes.

Acknowledgement. We thank G. Molchan for informing us about his work in 1960's, M. Taqqu for letting us know about Decreusefond and Üstünel 1997 and Samarov and Taqqu 1988, and L. Decreusefond and D. Feyel for the reference Feyel and de La Pradelle 1996. The first version of the present paper was completed before we became aware of this related and, as regards Molchan, much earlier work.

\section{References}

Decreusefond, L. and Üstünel, A. (1997). Stochastic analysis of the fractional Brownian motion. Potential Anal. To appear.

Feyel, D. and de La Pradelle, A. (1996). Fractional integrals and Brownian processes. Preprint, Université d'Évry.

Gripenberg, G. and Norros, I. (1996). On the prediction of fractional Brownian motion. J. Appl. Probab. 33, 400-410.

Huang, S. and Cambanis, S. (1978). Stochastic and multiple Wiener integrals for Gaussian processes. Ann. Probab. 6, 585-614.

Jacod, J. and Shiryaev, A. (1987). Limit Theorems for Stochastic Processes. Berlin: Springer.

Kolmogorov, A. (1940). Wienersche Spiralen und einige andere interessante Kurven im Hilbertschen Raum. C.R. (Doklady) Acad. Sci. USSR (N.S.) 26, 115-118.

Liptser, R. and Shiryaev, A. (1986). Theory of Martingales. Moscow: Nauka. In Russian.

Mandelbrot, B. and Van Ness, J. (1968). Fractional Brownian motions, fractional noises and applications. SIAM Rev. 10, 422-437.

Molchan, G. (1969). Gaussian processes with spectra which are asymptotically equivalent to a power of $\lambda$. Theory Probab. Appl. 14, 530-532.

Molchan, G. and Golosov, J. (1969). Gaussian stationary processes with asymptotic power spectrum. Soviet Math. Dokl. 10, No. 1. 
Revuz, D. and Yor, M. (1991). Continuous Martingales and Brownian Motion, volume 293 of Grundlehren der mathematischen Wissenschaften. Berlin: Springer.

Samarov, A. and Taqqu, M. (1988). On the efficiency of the sample mean in long memory noise. J. Time Ser. Anal. 9, 191-200. 